\title{
On EPR-type Entanglement in the Experiments of Scully et Al. II. Insight in the Real Random Delayed-choice Erasure Experiment
}

\author{
F. Herbut
}

\begin{abstract}
It was pointed out in the first part of this study [Herbut:Found. Phys. 38, 1046-1064 (2008)] that EPR-type entanglement is defined by the possibility of performing any of two mutually incompatible distant, i. e., direct-interaction-free, measurements. They go together under the term 'EPR-type disentanglement'. In this second part, quantum-mechanical insight is gained in the real random delayed-choice erasure experiment of Kim et al. [Kim et al.: Phys. Rev. Lett. 84, 1-5 (2000)] by a relative-realityof-unitarily-evolving-state (RRUES) approach (explained in the first part). Finally, it is shown that this remarkable experiment, which performs, by random choice, two incompatible measurements at the same time, is actually an EPR-type disentanglement experiment, closely related to the micromaser experiment discussed in the first part.
\end{abstract}

Keywords Real experiment. Delayed-choice erasure. Distant measurement. Random-choice EPR-type disentanglement. Detector as quantum mechanical system

F. Herbut (mail)

Serbian Academy of Sciences and Arts, Knez Mihajlova 35, 11000 Belgrade, Serbia 
e-mail: fedorh@sanu.ac.rs 


\section{Introduction}

Scully and Drühl published a thought experiment on erasure in 1982 [1], which, 18 years later Kim, Yu, Kulik, Shih, and Scully reported to have performed in an inessentially changed way [2]. In this article we investigate the experiment because it realizes several provoking and baffling fundamental quantum-mechanical ideas: (i) delayed choice (in the sense of Wheeler [3]), (ii) erasure, (iii) erasure in part of the state, (iv) delayed-choice era-

sure in the sense of Scully (or after-detection erasure), (v) random choice of particle-like or wave-like behavior after detection, and finally, (vi) EPR-type disentanglement.

The authors lean on Glauber's second-quantization theory for precise quantitative quantum mechanical predictions, which turn out well confirmed by the experiment. (For references to Glauber's theory and references to earlier work see the article of Kim et al. [2].)

One of the first attempts to perform a real erasure experiment [4] also presented its theoretical part in second quantization. However, it has turned out that first-quantization quantum mechanical insight [5] is feasible and useful.

A quantum mechanical analysis of the Kim et al. article [2] is presented in this paper in order to provide insight, shed more light, and help to demystify the mentioned puzzling quantum mechanical ideas. At last but not at least, this study, along with the preceding ones along parallel lines (Ref-s [5] and [6]), should hopefully help to understand in what direction one should look for an objective, preparation- and observation-independent quantum mechanics. 


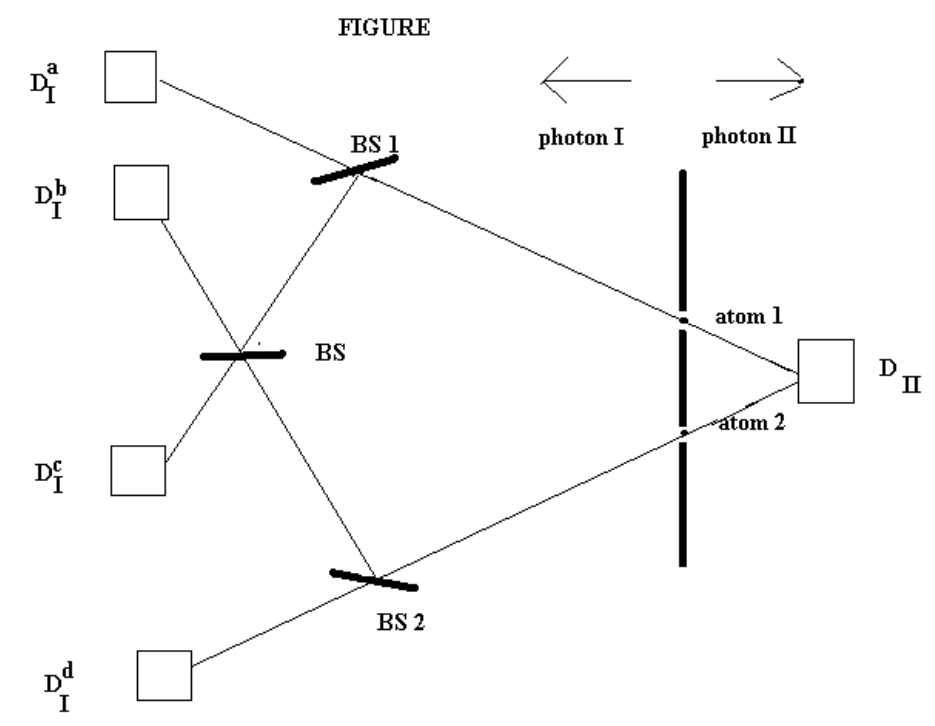

Figure 1: The two-photon 'two-slit' random-choice and delayed-choice erasure experiment outlined in section 2 .

\section{Basic Idea of the Kim et al. Experiment and Questions}

A quantum eraser experiment very close to and somewhat simpler in details than the experiment of Kim et al. [2] itself is illustrated in the Fig. (The notation is in accordance with that in the first part of this study [6].)

Two atoms labeled by 1 and 2 (counterparts of the two slits in Young's experiment [7]) are excited by a weak laser pulse. A pair of entangled quanta, photon $I$ and photon $I I$, is then emitted from either atom 1 or atom 2 (coherently added possibilities) by atomic cascade decay (emitting photons $I$ and $I I$ ). Photon $I I$, propagating to the right, is registered by detector $D_{I I}$, which can be scanned by a step motor along its $x$ axis for the observation of interference fringes. 
Photon $I$ propagates to the left. If the pair is generated in atom 1 , photon $I$ will follow path 1 (see the Fig.) meeting beam splitter $B S 1$ with $50 \%$ chance of being reflected or transmitted. If the pair is generated in atom 2 , photon $I$ will follow path 2 meeting the beam splitter $B S 2$ with $50 \%$ chance of being reflected or transmitted. In case of the $50 \%$ chance of being transmitted at either $B S 1$ or $B S 2$, photon $I$ is detected by either detector $D_{I}^{a}$ or $D_{I}^{d}$. The registration of $D_{I}^{a}$ or $D_{I}^{d}$ provides the which-path information (path 1 or path 2 respectively) of photon $I$, and this in turn provides the which-path information for photon $I I$ due to the entanglement nature of the two-photon state generated by the atomic cascade decay.

Given a reflection at either $B S 1$ or $B S 2$, photon $I$ continues its 1 or 2 path respectively to meet another (central) $50 \%-50 \%$ beam splitter $B S$, and then, possibilities 1 and 2 having interfered, photon $I$ is detected by either detectors $D_{I}^{b}$ or $D_{I}^{c}$ shown in the Fig. The triggering of detectors $D_{I}^{b}$ or $D_{I}^{c}$ erases the which-path information of photon II , and creates the which-coherence information.

The random choice takes place in the beam splitters $B S 1(2)$. As to the delayed-choice (in the sense of Scully), the which-path or both-path (random) choice in the beam splitters $B S 1$ and $B S 2$ is delayed compared to the detection of photon $I I$ in detector $D_{I I}$.

There are some questions that come to mind.

(i) If the quantum correlations in a bipartite state, like that of the twophoton system at issue, are such that one particle contains which-path information on the other, then there is no coherence in the single photon state (hence, one cannot detect interference) as it is well known. Can this be made 
obvious in the Kim et al. experiment?

(ii) It is known that pure-state entanglement is due to coherence in the state of the composite system, i. e., it stems from superposition of orthogonal uncorrelated bipartite states. How is the which-path information erased, and, particularly, how does the coherence descend from the bipartite system to the subsystem of photon- $I I$ ?

(iii) The behavior of the (improper [8]) ensemble of photons $I I$ is described by its state, i. e., by the reduced density operator $\rho_{I I}$, of photon $I I$. It is known that this state cannot be influenced by whatever happens to the photon- $I$ partner (improper) ensemble alone (as long as there is no interaction between the two photons). Can one see this in the description of the experiment?

(iv) Can a gradual increase in complexity of the concepts involved be displayed? There is the delayed choice in the sense of Wheeler [3], random choice between which-path and coherence information, Wheeler's delayed choice with erasure, and, finally, delayed choice in the sense of Scully with erasure in part of the state.

(v) It is known that quantum correlations can not provide signalling. This means that whatever happens to the individual photon $I$, no change is detectable on its partner, photon $I I$. How is erasure feasible in view of this fact?

(vi) Photon $I$ 'makes the delayed choice' by randomly being either transmitted in $B S 1(2)$ or being reflected there. The photon- $I I$ partner has before that been detected in detector $D_{I I}$. How can the one-path or interference origin of this localization be decided later, after the photon has been detected? 
Next we are going to give a precise first-quantization form to the above outline of a physical picture in order to enable one to answer the questions and gain generally more insight in the experiment.

\section{Quantum-mechanical Description}

In order to answer the questions posed, we transform now the verbal description of the experiment from the preceding section into a quantum mechanical two-photon state vector following the example of the simple quantum mechanical description of the Mach-Zehnder interferometer [9] (pp. 189-190).

One should have in mind that the "path" state of the photon is multiplied by the imaginary unit when reflected on a beam splitter, and is unchanged when transmitted (9], p. 189). Further, we denote by $|0\rangle_{D_{I I}}$ the state vector of the localization detector $D_{I I}$ of the second photon at the beginning of the experiment, and by $U_{I I D_{I I}}$ the unitary evolution operator containing the interaction between photon $I I$ and the localization detector in the process of localization and afterwards. Then, the two-photon state vector in the experiment, after passage of photon $I$ through the central beam splitter $B S$ (see the Fig.), but before it reaches any of the detectors $D_{I}^{q}, q=a, b, c, d$, having in mind the above outline of the experiment, reads as follows. (We write it down, then explain in detail.)

$$
\begin{gathered}
\left|(1,2) \rightarrow D_{I}^{a}, D_{I}^{b}, D_{I}^{c}, D_{I}^{d}\right\rangle_{I, I I}=(1 / 2)\left|1 \rightarrow D_{I}^{a}\right\rangle_{I}\left\{U_{I I D_{I I}}\left(|1\rangle_{I I}|0\rangle_{D_{I I}}\right)\right\}+ \\
(1 / 2)\left\{(1 / 2)^{1 / 2} i\left[i\left|1 \rightarrow D_{I}^{b}\right\rangle_{I}+\left|1 \rightarrow D_{I}^{c}\right\rangle_{I}\right]\right\}\left\{U_{I I D_{I I}}\left(|1\rangle_{I I}|0\rangle_{D_{I I}}\right)\right\}+ \\
(1 / 2)\left\{(1 / 2)^{1 / 2} i\left[\left|2 \rightarrow D_{I}^{b}\right\rangle_{I}+i\left|2 \rightarrow D_{I}^{c}\right\rangle_{I}\right]\right\}\left\{U_{I I D_{I I}}\left(|2\rangle_{I I}|0\rangle_{D_{I I}}\right)\right\}+
\end{gathered}
$$




$$
(1 / 2)\left|2 \rightarrow D_{I}^{d}\right\rangle_{I}\left\{U_{I I D_{I I}}\left(|2\rangle_{I I}|0\rangle_{D_{I I}}\right)\right\} .
$$

There are 4 (coherently added) possibilities for photon $I$ expressed by the rhs of (1). It may be emitted from atom 1 . Then it may be transmitted through or reflected from beam splitter BS1. These are the first two terms in (1). The last two terms cover the symmetric case: the possibility that photon $I$ is emitted from atom 2 .

In the moment of our description photon II has already been absorbed in detector $D_{I I}$, and the absorbed photon with the detector evolves (in some interacting way). Though, this seems to occur locally, i. e., independently of what happens to photon $I$, we must distinguish the two basic possibilities: photon II emitted by atom 1 and photon $I I$ emitted by atom 2 , because we make this distinction for photon $I$, and photons $I$ and II are emitted together (we disregard the small delay due to the cascade emission from the same atom). Hence, the first two terms in (1) have one and the same tensor factor $\left\{U_{I I D_{I I}}\left(|1\rangle_{I I}|0\rangle_{D_{I I}}\right)\right\}$, and the last two terms have the factor of the same form in which 1 is replaced by 2 .

The four possibilities exclude each other. Hence the terms are orthogonal. They are equally probable; each has probability $1 / 4$. The first term is the simplest because, having been transmitted through the beam splitter BS1, the spatial state vector $\left|1 \rightarrow D_{I}^{a}\right\rangle_{I}$ suffers no change of its phase factor.

The second term describes photon $I$ after it has passed the central beam splitter $B S$. The photon can be reflected from it. This gives $i \mid 1 \rightarrow$ $\left.D_{I}^{b}\right\rangle_{I}$. The other, equally probable, possibility is passing through $B S$, which results in $\left|1 \rightarrow D_{I}^{c}\right\rangle_{I}$. The two possibilities exclude each other (the corresponding terms are orthogonal) and they give the first-photon state vector in the large brackets in the second term of (1). It is multiplied by $i$ because the two possibilities in it take place after reflection from $B S 1$. 
As mentioned, the third and fourth terms describe the symmetric cases stemming from the (coherently added) possibility that photon $I$ was emitted from the second atom.

Actually, there should also be the (coherently added) possibility that photon $I$ misses the beam splitters $B S 1(2)$ etc. But this component of the bipartite state vector is left out (projected out) because it is irrelevant in the experiment.

To answer the questions from the preceding section, we need, besides the state vector (1), also the state (reduced density operator) of photon II . This is easy to evaluate if one rewrites (1) in the form of an expansion in an ortho-normal basis for photon $I$. But first, we can simplify (1).

One actually has $\left|1 \rightarrow D_{I}^{r}\right\rangle_{I}=\left|2 \rightarrow D_{I}^{r}\right\rangle_{I}, r=b, c$ because, after passage through the central beam splitter, photon $I$ cannot 'remember' where it has come from. Therefore, we can write instead of $\left|q \rightarrow D_{I}^{r}\right\rangle_{I} q=$ $1,2 r=b, c \quad\left|\rightarrow D_{I}^{r}\right\rangle_{I}$. One can also replace $\left|1 \rightarrow D_{I}^{a}\right\rangle_{I}$ and $\left|2 \rightarrow D_{I}^{d}\right\rangle_{I}$ by $\left|\rightarrow D_{I}^{a}\right\rangle_{I}$ and $\left|\rightarrow D_{I}^{d}\right\rangle \quad$ respectively because detector $D_{I}^{a}$ can be reached only if photon $I$ is emitted from atom 1 and symmetrically (we omit the redundant information).

After omission of $1(2)$ in the state vector of photon $I$ in (1) as explained, relation (1) can be rearranged so that we have an expansion in the ortho-normal basis $\left\{\left|\rightarrow D_{I}^{r}\right\rangle_{I}: r=a, b, c, d\right\}$ of photon $I$ as easily seen.

$$
\begin{gathered}
\left|(1,2) \rightarrow D_{I}^{a}, D_{I}^{b}, D_{I}^{c}, D_{I}^{d}\right\rangle_{I, I I}= \\
(1 / 2)\left|\rightarrow D_{I}^{a}\right\rangle_{I}\left\{U_{I I D_{I I}}\left(|1\rangle_{I I}|0\rangle_{D_{I I}}\right)\right\}+ \\
(1 / 2)\left|\rightarrow D_{I}^{b}\right\rangle_{I} U_{I I D_{I I}}\left\{\left[(1 / 2)^{1 / 2}\left(-|1\rangle_{I I}+i|2\rangle_{I I}\right)\right]|0\rangle_{D_{I I}}\right\}+
\end{gathered}
$$




$$
\begin{gathered}
(1 / 2)\left|\rightarrow D_{I}^{c}\right\rangle_{I} U_{I I D_{I I}}\left\{\left[(1 / 2)^{1 / 2}\left(i|1\rangle_{I I}-|2\rangle_{I I}\right)\right]|0\rangle_{D_{I I}}\right\}+ \\
(1 / 2)\left|\rightarrow D_{I}^{d}\right\rangle_{I}\left\{U_{I I D_{I I}}\left(|2\rangle_{I I}|0\rangle_{D_{I I}}\right)\right\} .
\end{gathered}
$$

The state (reduced density operator)

$$
\rho_{I I} \equiv \operatorname{tr}_{I}\left(\left|(1,2) \rightarrow D_{I}^{a}, D_{I}^{b}, D_{I}^{c}, D_{I}^{d}\right\rangle_{I, I I}\left\langle(1,2) \rightarrow D_{I}^{a}, D_{I}^{b}, D_{I}^{c},\left.D_{I}^{d}\right|_{I, I I}\right)\right.
$$

of photon II can now easily be evaluated from (2) (because all 'off-diagonal' terms give zero due to the orthogonality of the first-photon basis).

$$
\begin{aligned}
\rho_{I I}= & (1 / 4)\left\{U_{I I D_{I I}}\left(|1\rangle_{I I}|0\rangle_{D_{I I}}\right)\right\}\left\{\left(\left\langle\left. 1\right|_{I I}\left\langle\left. 0\right|_{D_{I I}}\right) U_{I I D_{I I}}^{\dagger}\right\}+\right.\right. \\
& (1 / 4) U_{I I D_{I I}}\left\{\left[(1 / 2)^{1 / 2}\left(-|1\rangle_{I I}+i|2\rangle_{I I}\right)\right]|0\rangle_{D_{I I}}\right\} \times \\
& {\left[( 1 / 2 ) ^ { 1 / 2 } \left(-\left\langle\left. 1\right|_{I I}-i\left\langle\left. 2\right|_{I I}\right)\right]\left\langle\left. 0\right|_{D_{I I}}\right\} U_{I I D_{I I}}^{\dagger}+\right.\right.} \\
& \left.(1 / 4) U_{I I D_{I I}}\left\{\left[(1 / 2)^{1 / 2}\left(\left.i\right|_{1}\right\rangle_{I I}-|2\rangle_{I I}\right)\right]|0\rangle_{D_{I I}}\right\} \times \\
& \left\{\left[( 1 / 2 ) ^ { 1 / 2 } \left(-i\left\langle\left. 1\right|_{I I}-\left\langle\left. 2\right|_{I I}\right)\right]\left\langle\left. 0\right|_{D_{I I}}\right\} U_{I I D_{I I}}^{\dagger}+\right.\right.\right. \\
& (1 / 4)\left\{U_{I I D_{I I}}\left(|2\rangle_{I I}|0\rangle_{D_{I I}}\right)\right\}\left\{\left(\left\langle\left. 2\right|_{I I}\left\langle\left. 0\right|_{D_{I I}}\right) U_{I I D_{I I}}^{\dagger}\right\} .\right.\right.
\end{aligned}
$$

Multiplying out the terms, one obtains

$$
\begin{gathered}
\rho_{I I}=(1 / 2)\left\{U_{I I D_{I I}}\left(|1\rangle_{I I}|0\rangle_{D_{I I}}\right)\right\}\left\{\left\langle\left. 1\right|_{I I}\left\langle\left. 0\right|_{D_{I I}}\right)\right\} U_{I I D_{I I}}^{\dagger}+\right. \\
(1 / 2)\left\{U_{I I D_{I I}}\left(|2\rangle_{I I}|0\rangle_{D_{I I}}\right)\right\}\left\{\left(\left\langle\left. 2\right|_{I I}\left\langle\left. 0\right|_{D_{I I}}\right) U_{I I D_{I I}}^{\dagger}\right\} .\right.\right.
\end{gathered}
$$

The time variable in the unitary evolution operator $U_{I I D_{I I}}$ has been suppressed. As it has been stated, its value is some instant after photon $I$ has passed the central beam splitter $B S$, but has not yet reached any of 
the four detectors $D_{I^{-}}^{a} D_{I}^{d}$ (or, possibly, has already been detected in the first or in the last of them).

If the experiment were a simple erasure (before-detection) one, then one would still have lack of interaction between photon $I I$ and the localization detector $D_{I I}$ at the moment in question: $U_{I I D_{I I}}=U_{I I} U_{D_{I I}}$. Hence, detector $D_{I I}$ could be omitted (moved from the 'object' of description to the 'subject'). If, on the contrary, we have delayed-choice (or after-detection) erasure, then we have interaction, and the description as it stands is essential (cf insight in delayed-choice erasure in part I [6]).

One should note that the four terms in (1) are coherently mixed (the terms are superposed). It is of crucial importance to preserve this coherence during the experiment. Nowadays much thinking goes on about the problem how to counteract decoherence (see e. g. [10]), which destroys coherence.

\section{Answers}

Relations (1) and (4) give answer to question (i). The former makes it obvious that orthogonal states of photon $I$ in the first two terms on the one hand and the last two terms on the other 'mark' or distinguish the 'being emitted from atom 1' and 'being emitted from atom 2' respective states of photon II (latent 'which-path' information). Relation (4) then makes it evident that this has the consequence of 'suppressing' the coherence in the state of photon $I I$.

Note that the much-used term 'erasure' is not meant to be a synonym for this 'suppression'. On the contrary, it denotes elimination of the described 
mechanism of 'suppression', which is still present in (1) or (2). The very detection of the beam of photons $I$ in the detectors $D_{I}^{r} r=a, b, c, d$, turns (2) into a (proper) mixture (of the 4 component states in (2)). Then, also $\rho_{I I}$ given by (3) is a proper mixture containing two coherence states (terms 2 and 3 ).

Answer to question (ii) follows from the fact that the central beam splitter $B S$ (cf the Fig.) treats the components of photon $I$ coming from atoms 1 and 2 differently only by a phase difference of $(\pi / 2)$ (multiplication by $i$ ). This makes possible erasure, so that, upon detection in $D_{I}^{r}, r=a, b, c, d$, half of the ensemble of photons $I I$ displays interference (cf relation $(3)$ ).

Note that state decomposition (3), as it stands, is only a mathematical relation. The second and third subensembles of the ensemble of photons II in it are not defined physically on the local, photon-II, level. They are defined only distantly (or, one might say, globally) on part of photon $I$ propagating towards the detectors $D_{I}^{r}, \quad r=b, c$.

Question (iii) is answered in the affirmative by comparing relations (2) and (4): in spite of the erasure in part of the two-photon state vector displayed in (2), locally, i. e., in the entire ensemble of photons $I I$, there is no change induced (as obvious in (4)).

To answer question (iv), we can begin by drastically downgrading the Kim et al. experiment, and then by upgrading it in steps.

a). Let us imagine that one, so to say, 'by hand' either just removes the beam splitters $B S 1(2)$ or, as an alternative, replaces them by mirrors. 
Further, we imagine that the choice between these two possibilities is made after the photons begin to propagate from the atoms (the 'slits'). Thus, the choice of a particle-like or a wave-like experiment would be delayed with respect to the moment of preparation (or beginning of the experiment).

This would constitute a genuine Wheeler delayed-choice experiment (though upgraded from the original one-photon case [3] to a two-photon one).

b). In the Kim et al. experiment the 'by hand' choice is replaced by a random mechanism (transmission or reflection on the beam splitters BS1(2)). This upgrading of Wheeler's idea is extremely important on two counts:

(i) Let us remember the famous wave-particle duality form of Bohr's complementarity principle and the rebellions against its claim of universal validity (see the impressive work of Ghose and Home in [11], and the references therein). The random choice mingles particle-like and wave-like behavior in one experiment, thus giving support to the mentioned rebellions.

(ii) At the very beginning of the first erasure paper of Scully et al. [1] the authors say:

"... the role of the observer lies at the heart of the problem of measurement and state reduction in quantum mechanics." (Italics by F. H.)

A few lines lower they say:

"... Wheeler has pointed out that the experimentalist may delay his decision as to display wave-like or particle-like behavior in a light beam long after the beam has been split by the appropriate optics." (Italics by F. H.)

The random-choice property of the Kim et al. experiment (beam splitters $B S 1(2)$ ) has replaced the 'decision' of the 'observer' or the 'experimenter' 
by an automatic step in the experiment. This goes a long way in warning against the fallacy of overestimating the role of the human observer.

The Kim et al. experiment performs a partial-state erasure having the two-photon state in mind. This is not partial erasure. The erasure is complete, but it takes place only in a part of the two-photon state. Since states are ensembles in the laboratory, one experimentally deals with two subensembles: the one 'seen' in the coincidence of the detectors $D_{I}^{r}$ and $D_{I I}$, $r=a, d$, ('which path') and the other 'seen' in the $D_{I}^{r}, D_{I I}, r=b, c$ coincidence ('which interference').

c) Kim et al. actually performed a delayed-choice (in the sense of Scully) or after-detection (of photon $I I$ ) experiment in which this detection was performed so early in the experiment, that photon $I$ has not yet reached the beam splitters $B S 1(2)$, i. e., the random choice has not yet taken place. (This seems to put a dramatic emphasis on question vi).)

I believe that the answer to question (v) has now become clear. The fact that no signal can be sent from photon $I$ to photon $I I$ is based on the circumstance that the entire state (ensemble) $\rho_{I I}$ of photons $I I$ (cf (4)) cannot be distantly manipulated on account of entanglement. Whatever change photon $I$ undergoes due to some local interaction, this does not influence the state $\rho_{I I}$ of photon $I I$. But this is not so if one makes coincidence measurements on photon $I$ and photon $I I$. Then distant (or global) influence does show up and erasure can appear.

Answer to question (vi) was given in part I [6]: the combined photon$I I-D_{I I}$ system is analogous to the photon- $I I$ system alone as far as the definite-way or coherence states are concerned. Therefore, there is no ques- 
tion of 'acting backwards in time' or any other mystification.

\section{The Experiment is an EPR-type Disentanglement}

Now we proceed to a deeper layer of physical insight in the random-choice and delayed-choice erasure experiment that we investigate: we view it as an EPR-type disentanglement in which both mutually incompatible distant measurements are performed simultaneously.

The random EPR-type disentanglement interpretation of the experiment can, actually, be seen from (2) and (3). Namely, it is evident that the first and the last terms in both equations refer to distant 'which-path' relation, and the second and third terms in them describe 'which-interference' relations, both in a latent way until the corresponding detector is reached. It is clear from (3) and (2) that the improper ensemble of photons II is seen as broken up into the mentioned two (improper) sub-ensembles distantly, i. e., due to a relevant difference in the photon- $I$ - partners. Locally, each individual photon $I I$ is in the same state $\rho_{I I}$ given by (4).

This decomposition of the ensemble is of the type $\rho_{I I}=(1 / 2) \rho_{I I}+$ $(1 / 2) \rho_{I I}$. It is one of the beauties of the experiment. Namely, both the 'which-path' part and the 'which-interference' part, taken separately, look like the two separate experiments which make up the EPR-type disentan-

glement, and which are in most other experiments performed alternatively at the will of the experimenter. Here both experiments are performed together on account of the random-choice function of the first beam splitters $B S q, q=1,2$. 
It is also worth mentioning that one has two interferences

$$
(1 / 2)^{1 / 2}\left[-U_{I I D_{I I}}\left(|1\rangle_{I I}|0\rangle_{D_{I I}}\right)+i U_{I I D_{I I}}\left(|2\rangle_{I I}|0\rangle_{D_{I I}}\right)\right]
$$

and

$$
(1 / 2)^{1 / 2}\left[i U_{I I D_{I I}}\left(|1\rangle_{I I}|0\rangle_{D_{I I}}\right)-U_{I I D_{I I}}\left(|2\rangle_{I I}|0\rangle_{D_{I I}}\right)\right]
$$

( $\operatorname{cf}(2)$ ), which are 'opposite' in the sense that the corresponding (pure-state) density matrices add up into $\rho_{I I}$ given by (4) (cf the second and third terms in (3), which add up into (4)).

All that remains to be done is to prove a formal claim made in the first part [6] of this study. It was shown there that there are two simple coherence bases (in the Schmidt canonical expansion relevant for EPR-type disentanglement) $| \pm\rangle_{I} \equiv(1 / 2)^{1 / 2}\left(|1\rangle_{I} \pm|2\rangle_{I}\right) \quad$ (equation (10) there) and $| \pm i\rangle_{I} \equiv(1 / 2)^{1 / 2}\left(|1\rangle_{I} \pm i|2\rangle_{I}\right) \quad$ (equation (11) there). It was, further, shown that the first simple coherence bases are realized in the micro-maser experiment [12], and it was claimed that the second simple coherence basis finds realization in the experiment of Kim et al. that is the object of investigation in this article.

If we project out and renormalize the 'which-coherence' part in (2), and move some numerical factors from the second tensor factor to the first, we obtain

$$
\begin{gathered}
\left|(1,2) \rightarrow D_{I}^{b}, D_{I}^{c}\right\rangle_{I, I I}= \\
(1 / 2)^{1 / 2}\left(-\left|\rightarrow D_{I}^{b}\right\rangle_{I} U_{I I D_{I I}}\left\{\left[(1 / 2)^{1 / 2}\left(|1\rangle_{I I}-i|2\rangle_{I I}\right)\right]|0\rangle_{D_{I I}}\right\}+\right. \\
\left.i\left|\rightarrow D_{I}^{c}\right\rangle_{I} U_{I I D_{I I}}\left\{\left[(1 / 2)^{1 / 2}\left(|1\rangle_{I I}+i|2\rangle_{I I}\right)\right]|0\rangle_{D_{I I}}\right\}\right) .
\end{gathered}
$$

Next we find out how to express the orthonormal basis vectors $-\left|\rightarrow D_{i}^{b}\right\rangle_{I}$, $i\left|\rightarrow D_{I}^{c}\right\rangle_{I}$ appearing in (5) in terms of the state vectors $|1\rangle_{I},|2\rangle_{I}$, which 
(by definition) denote the component states of photon $I$ propagating from the respective first beam splitters $B S q, q=1,2$, towards the central beam splitter $B S$ (cf the Figure). The state vectors $|1\rangle_{I},|2\rangle_{I}$ are the normalized relevant projections of the initial corresponding state vectors expressing propagation from the atoms to the first beam splitters. Their definition corresponds to that of the coherence component (5) of the two-photon state vector, which is analogously projected out (and renormalized) from (2).

Let the unitary operator $U_{I}$ stand for the evolution of the first photon from these component state vectors $|q\rangle_{I}, q=1,2$, to the respective states that appear after passing the central beam splitter $B S$ (where $\left|\rightarrow D_{I}^{r}\right\rangle, \quad r=b, c$ are defined).

We make use of the equality $\left|1 \rightarrow D_{I}^{r}\right\rangle_{I}=\left|2 \rightarrow D_{I}^{r}\right\rangle_{I}, r=1,2 \quad(\mathrm{cf}$ beneath (1)) again. Then, one can see from relation (1) that

$$
\begin{gathered}
U_{I}|1\rangle_{I}=(1 / 2)^{1 / 2}\left(-\left|\rightarrow D_{I}^{b}\right\rangle_{I}+i\left|\rightarrow D_{I}^{c}\right\rangle_{I}\right), \\
U_{I}|2\rangle_{I}=(1 / 2)^{1 / 2}\left(i\left|\rightarrow D_{I}^{b}\right\rangle_{I}-\left|\rightarrow D_{I}^{c}\right\rangle_{I}\right),
\end{gathered}
$$

Solving (6a) and (6b) for $\left|\rightarrow D_{I}^{r}\right\rangle_{I}, \quad r=b, c$, one obtains

$$
-\left|\rightarrow D_{I}^{b}\right\rangle_{I}=(1 / 2)^{1 / 2}\left[\left(U_{I}|1\rangle_{I}\right)+i\left(U_{I}|2\rangle_{I}\right)\right]
$$

and

$$
i\left|\rightarrow D_{I}^{c}\right\rangle_{I}=(1 / 2)^{1 / 2}\left[\left(U_{I}|1\rangle_{I}\right)-i\left(U_{I}|2\rangle_{I}\right)\right] .
$$

If we replace in (5) the basis vectors $-\left|\rightarrow D_{i}^{b}\right\rangle_{I}, \quad i\left|\rightarrow D_{I}^{c}\right\rangle_{I}$ with the expressions given by (7a) and (7b) respectively, we obtain

$$
\begin{gathered}
\left|(1,2) \rightarrow D_{I}^{b}, D_{I}^{c}\right\rangle_{I, I I}=\left\{(1 / 2)^{1 / 2}\left[\left(U_{I}|1\rangle_{I}\right)+i\left(U_{I}|2\rangle_{I}\right)\right]\right\} \otimes \\
\left\{(1 / 2)^{1 / 2}\left[\left(U_{I I D_{I I}}\left(|1\rangle_{I I}|0\rangle_{D_{I I}}\right)\right)-i\left(U_{I I D_{I I}}\left(|2\rangle_{I I}|0\rangle_{D_{I I}}\right)\right)\right]\right\}+
\end{gathered}
$$




$$
\begin{gathered}
\left\{(1 / 2)^{1 / 2}\left[\left(U_{I}|1\rangle_{I}\right)-i\left(U_{I}|2\rangle_{I}\right)\right]\right\} \otimes \\
\left.\left\{(1 / 2)^{1 / 2}\left[\left(U_{I I D_{I I}}\left(|1\rangle_{I I}\right)|0\rangle_{D_{I I}}\right)\right)+i\left(U_{I I D_{I I}}\left(|2\rangle_{I I}|0\rangle_{D_{I I}}\right)\right)\right]\right\} .
\end{gathered}
$$

Equation (8) is a Schmidt canonical expansion, essentially, in the mentioned second simple basis $| \pm i\rangle_{I} \equiv(1 / 2)^{1 / 2}\left(|1\rangle_{I} \pm i|2\rangle_{I}\right)$.

To understand better the state vectors appearing in (8), we write down also the 'which-path' part of (2). For comparison with (8), let us introduce the state vectors $|q\rangle_{I}^{\prime}, q=1,2$, as the components that are transmitted through the beam splitters $B S q$, and for their further evolution we utilize $U_{I}^{\prime}$, under the action of which they become $\left|\rightarrow D_{I}^{r}\right\rangle_{I}, \quad r=a, d$ respectively. We obtain

$$
\begin{gathered}
\left|(1,2) \rightarrow D_{I}^{a}, D_{I}^{d}\right\rangle_{I, I I}=\left(U_{I}^{\prime}|1\rangle_{I}^{\prime}\right)\left(U_{I I D_{I I}}\left(|1\rangle_{I I}|0\rangle_{D_{I I}}\right)\right)+ \\
\left(U_{I}^{\prime}|2\rangle_{I}^{\prime}\right)\left(U_{I I D_{I I}}\left(|2\rangle_{I I}|0\rangle_{D_{I I}}\right)\right) .
\end{gathered}
$$

If the lhs of (8) would equal that of (9), and if one could drop the prims in (9), i. e., if one could write $U_{I}^{\prime}|q\rangle_{I}^{\prime}=U_{I}|q\rangle_{I}, \quad q=1,2$, then (8) and (9) would be a true parallel to the 'which-path' and 'which-interference' EPRtype disentanglement in the micromaser discussed in part I [6], only that instead of the first, we would have the second simplest basis (as explained above).

In the real experiment that we are discussing one must pay a price for having the two complementary disentanglements in one experiment. Namely, one must by projection and renormalization decompose (2) into (9) and (8) because they are two distinct parts of the same experiment (not two versions). Correspondingly, one has $|q\rangle_{I}^{\prime} \neq|q\rangle_{I}, \quad q=1,2$ on account of the two distinct projections (and renormalizations) of the component states. 
Nevertheless, having these peculiarities in mind, the claim from part I has been shown to be, essentially, valid. 


\section{Concluding Remarks}

The experiment discussed in this article provides us with a clear understanding of the distinction of 'potential' and 'actual' in the usual sense of these words (cf remark $\mathrm{F}$ in the first part of this study [6]).

Let us think of the experiment at issue as if it were performed in the photon-by-photon version. Then all the coherent possibilities (terms in (1) or (2)) are the photon's realities though still in a relative sense with respect to the preparator. We call them 'potential' with respect to our subjective choices of highlighting parts of it. 'Actuality' comes to the fore when we consider a detection coincidence, e. g., $D_{I}^{b}$ and $D_{I I}^{n} \quad$ (see part I [6] for

$\left.D_{I I}^{n}\right)$. Then, taking photon by photon, and considering different values of $n$, the fringes of the corresponding interference pattern come about. But this is no more than a subjective highlighting of part of the reality of the experiment. The single photon in reality has both the which-path property and the coherence property. (And by this it has both which-path and both coherence possibilities.)

This is analogous to the original EPR situation [13] with position and linear momentum disentanglement. But the present experiment has a big mentioned advantage. The original EPR (thought) experiment could have this advantage only if it contained measurement of position and momentum in the same experiment.

The Kim et al. experiment is, in the opinion of the present author, perhaps the most accomplished realization of an EPR-type disentanglement. I believe that this experiment is so important from the foundational point of view that it should be performed both in the photon-by-photon version, like 
e. g. the Mach-Zehnder interferometer experiments [14], [15], and in terms of positive-rest-mass particles.

Jaynes writes [16] (the last passage in the web version):

$"$... it is pretty clear why present quantum theory not only does not use - it does not even dare to mention - the notion of a "real physical situation". Defenders of the theory say that this notion is philosophically naive, a throwback to outmoded ways of thinking, and that recognition of this constitutes deep new wisdom about the nature of human knowledge. I say that it constitutes a violent irrationality, that somewhere in this theory the distinction between reality and our knowledge of reality has become lost, and the result has more the character of medieval necromancy than of science. It has been my hope that quantum optics, with its vast new technological capability, might be able to provide the experimental clue that will show how to resolve these contradictions."

Isn't it possible that Jaynes' hope has, at least to some extent, come true precisely on account of the work of Scully et al.? I find it hard to think of the Kim et al. experiment [2] in any other terms than as a "real physical situation". Its comprehension suggests the RRUES interpretation ( see part I). Then why not think of reality, at least as far as experiments are concerned, in this way? Understanding experiments is the natural springboard for understanding nature.

ACKNOWLEDGEMENTS The author is indebted to the unknown ref- 
erees who, besides useful suggestions, also helped substantially to achieve a presentation that is more readable.

\section{References}

[1] Scully, M. O., Drühl, K.: Quantum eraser: A proposed photon correlation experiment concerning observation and "delayed-choice" in quantum mechanics. Phys. Rev. A 25, 2208-2213 (1982)

[2] Kim Y.-H., Yu R., Kulik S. P., Shih Y., Scully M. O.: Delayed "choice" quantum eraser. Phys. Rev. Lett. 84, 1-5 (2000)

[3] Wheeler J. A.: The 'past' and the delayed-choice double-slit experiment. In: Marlow A. R. ed. Mathematical Foundations of Quantum Theory. Academic Press, New York (1978), pp. 9-48

[4] Kwiat P. G., Steinberg A. M., Chiao R. Y.: Observation of a "Quantum Eraser": A Revival of Coherence in a Two-photon Interference Experiment. Phys. Rev. A 45, 77297739 (1992)

[5] Herbut F., Vujičić M.: First-quantisation quantum-mechanical insight into the Hong-Ou-Mandel two-photon interferometer with polarizers and its role as a quantum eraser. Phys. Rev. A 56, 1-5 (1997)

[6] Herbut F.: On EPR-type Entanglement in the Experiments of Scully et al.I The Micromaser Case and Delayed-Choice Quantum Erasure. Found. Phys. 38, 1046-1064 (2008). ArXiv:quant-ph/0808.3176

[7] T. Young, Trans. R. Soc. XCII 12, 387 (1802). A quantum-mechanical discussion in [17] 
[8] D'Espagnat B.: Conceptual Foundations of Quantum Mechanics. Second Edition. W. A. Benjamin, Inc., Reading, Massachusetts (1976). Subsection 7.2

[9] Bub J.: Interpreting the Quantum World. Cambridge University Press, N. Y. (1997)

[10] Agarwal, G.S., Scully, M.O., Walther, H: Inhibition of Decoherence due to Decay in a Continuum. Phys. Rev. Lett. 86, 4271-4274 (2001)

[11] Ghose P., Home D.: The two-prism experiment and wave-aprticle duality of light. Found. Phys. 26, 943-953 (1996)

[12] Scully, M.O., Englert B.-G., Walther, H.: Quantum optical tests of complementarity. Nature 351, 111-116 (1991)

[13] Einstein A., Podolsky B., Rosen N.: Can quantum-mechanical description of physical reality be considered complete? Phys. Rev. 47, 777-780 (1935)

[14] Grangier P., Roger G., Aspect A.: Experimental evidence for a photon anricorrelation effect on a beam splitter: A new light on single-photon interferences. Europhys. Lett. 1, 173-179 (1986)

[15] Jacques V., Wu E., Grosshans F., Treussart F.,Grangier P., Aspect A., Roch J.-F.: Delayed-choice test of complementarity with single photons. Archiv quant-ph/0801.0979

[16] Jaynes, E. T.: in Foundation of Radiation Theory and Quantum Electronics, Barut A. ed., Plenum, 1980; also item 38 of the Jaynes bibliography on the web 
[17] Herbut F.: Quantum interference viewed in the framework of probability theory. Am. J. Phys. 60, 146-150 (1992) 